\title{
Estudio del Diseño de Talud de las Escombreras Mineras para Mitigar el Impacto Ambiental por Deslizamiento
}

\section{Slope Design Study of the Mining Tailings to Mitigate the Environmental Impact by Sliding}

${ }^{1}$ Carlos Huisa Ceori. ${ }^{2}$ Zoilo Gualberto Tejada Bedoya, ${ }^{3}$ Zenón Sarmiento Mejía

\begin{abstract}
RESUMEN:
Schuster y Kockelman proponen una serie de principios generales y metodologias para la reducción de amenazas de deslizamiento utilizando sistemas de prevención. los cuales requieren de políticas del Estado y de colaboración y conciencia de la empresa Minera. La estabilización de deslizamientos activos o potencialmente inestables es un trabajo relativamente complejo, el cual requiere de metodologías de diseño y construcción. En el presente trabajo se demuestra que los botaderos de la mina (uajone son materiales inestables tal como se ha modelado donde F.S. son menores a 01 y con el monitoreo del talud del botadero $N^{\circ} 05$ de la zona Inchupampa el desplazamiento total, horizontal y vertical es $2.9 .3 .091 .3 .9 \mathrm{~cm}$ dia.
\end{abstract}

Palabras Clave; escombrera, relieve, topografia, talud. geotecnia, deslizamiento, impacto ambiental

\section{ABSTRACT:}

Schuster and Kockelman. propose a set of general principles and methodologies to reduce threat sliding using prevention sysems, which require of state policies, collaboration and awarenew of the mining company. The stabilizalion of active slidings or potentially unstable is a relatively complex work, which requires of design and construction methodologies. The present study demonstrates that the dumps from Cuajone mine are unstahle materials that as it has been showed where FS is lower than 01 and with the dump slope monitoring No 05 the area of Inchupampa the total displacement. horizontal and vertical is 2.9 .3 .09 and $3.9 \mathrm{~cm} / \mathrm{day}$.

Keywords: dump, relief, topography; slope, geotechnical, landslide, environmental impact.

\footnotetext{
Masier Sciencie en Tecnologia Educativa. Ingeniero de Minas. Facultad de Ingenieria. Universidad Nacional Jorge Basadre Grohmann

${ }^{2}$ Ingeniero Geólogo. Facultad de Ingenieria. Universidad Nacional Jorge Basadre Grohmann

${ }^{3}$ Ingeniero de Minas. Facuitad de Ingenieria. Universidad Nacional Jorge Basadre Grohmann
} 


\section{INTRODUCCIÓN}

La explotación mediante el tipo de minado a tajo o cielo abierto, esta determinado por la cantidad de material que se tiene que mover, que se inicia en la etapa de la preparación del área para la construcción del tajo, removiendo gran cantidad de material para acceder al mineral; la remoción de los volúmenes pueden demorar años antes de que la unidad entre en producción. La segunda etapa consiste en extraer el mineral junto con el desmonte que en algunas minas supera el $100 \%$ al mineral llamado riqueza o de alta ley, Todo este desmonte considerado de muy baja ley o sin valor económico que para las empresas es un gasto desfavorable mover grandes cantidades y para compensar en algo estos gastos tratan de ubicarlos en lugares más cercano al tajo buscando relieves que sean favorables en la deposición o sea favorezcan al volteo de la tolva de los camiones. Estos hechos en gran medida modifican el relieve; la topografía, y geomorfología del lugar, la dimensión del minado va determinar el grado de impacto y el cambio del paisaje producido.

En la mina Cuajone los desmontes están siendo transportados cerca al botadero Torata Este. Los botaderos están extendiéndose actualmente por simple descarga de volquetes desde la cresta resultando en capas simples de descarga con alturas de hasta $200 \mathrm{~m}$.

Los botaderos futuros (y expansiones futuras de Torata Oeste y Torata Este) se construirán similarmente como capas simples de descarga para rellenar los valles. En Torata Oeste se planean tentativamente capas múltiples de $30 \mathrm{~m}$ encima del botadero existente, para acomodar mejor la operación de lixiviación. Se asume un ángulo de reposo de 37 grados en los diseños. El desmonte proveniente del desbroce de material de la pared este durante las diferentes fases de minado se transportará en mientras el desbroce de la pared sur utilizara el botadero Cocotea Oeste. El material lixiviable se continuará enviando al botadero Torata Oeste. Cuajone Este y Torata Este tomarán el desmonte de la pared norte y oeste según los programas de desbroce. En la Figura $\mathrm{N}^{\circ} 01$ se puede apreciar la ubicación de los botaderos.

\section{Descripción del Problema}

El cambio del paisaje se aprecia a simple vista, modificando el relieve; la topografia, y geomorfología del lugar, la dimensión del minado va determinar-el grado de impacto y el cambio del paisaje producido.

Según los autores (Vaughan (1989.), Elizondo (1994), los principales impactos ambientales causados por la minería a cielo abierto son los siguientes:

Afectación de la superficie: Devasta la superficie, modifica severamente la morfología del terreno, apila y deja al descubierto grandes cantidades de material estéril, produce la destrucción de áreas cultivàđas y de otros patrimonios superficiales, puede alterar ctrsos de aguas y formar grandes lagunas para el material descartado,

Afectación del entorno en general: Transforma radicalmente el entorno, pierde su posible atracción escénica y se ve afectado.

De esta gama de impactos nace la siguiente interrogante de investigación:

¿El diseño de la pendiente (Talud) de las pilas de desmonte podrá garantizar las medidas de seguridad preventivas, y una adecuada colocación de los materiales para mitigar el impacto ambiental?

\section{ULMETODOLOGÍA DELANNVESTIGACIÓN}

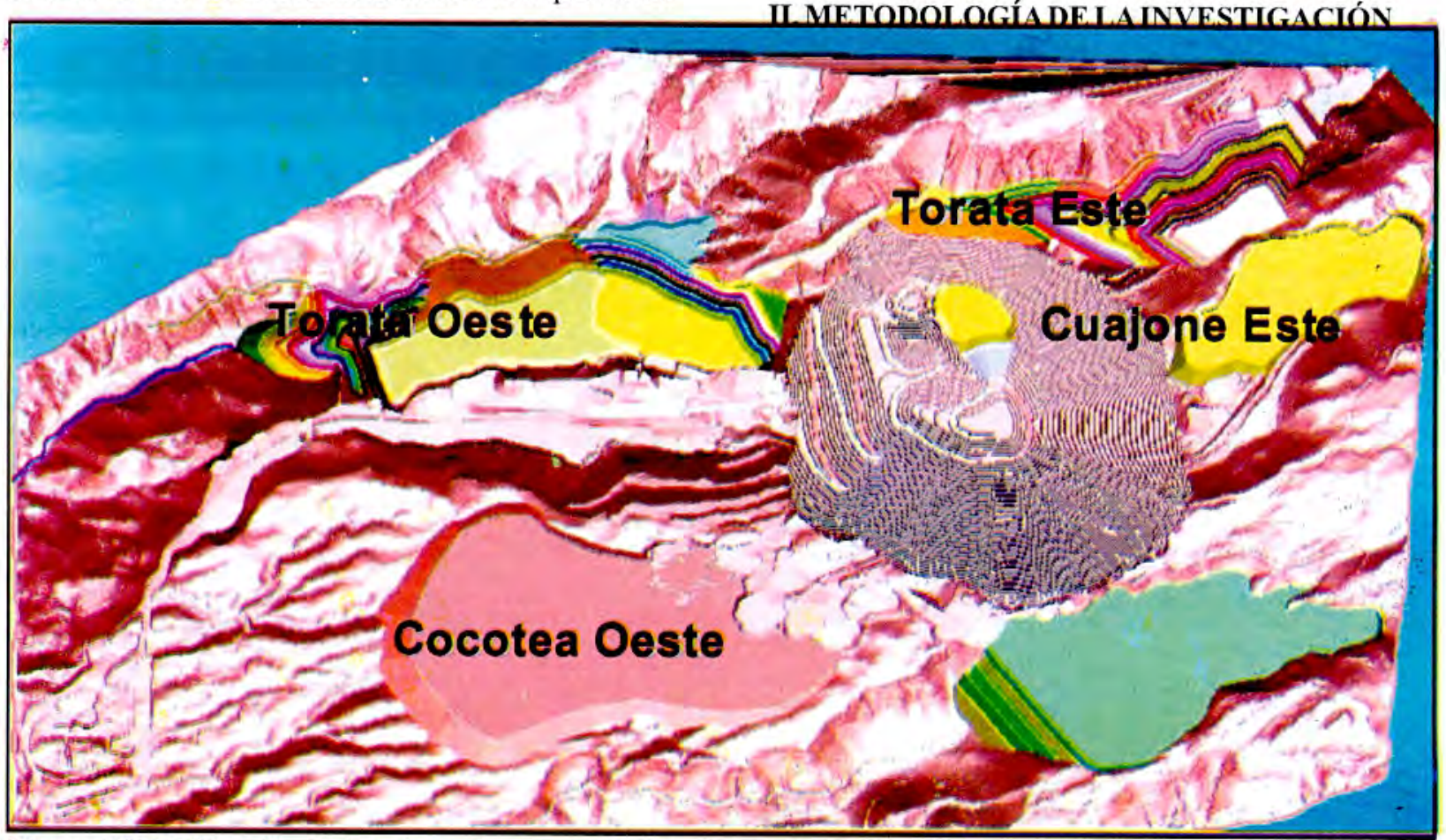

Figura N001:Ubicación de botaderos de desmonte. Bot. Torata Oeste Este, Cuajone Este, Cocotea Este y Oeste.

Fuente: Departamento de Geologia de Cuajone 


\section{METODOLOGÍADE LA INVESTIGACIÓN}

En el siguiente esquema se traduce la metodología utilizada en el presente trabajo de investigación.

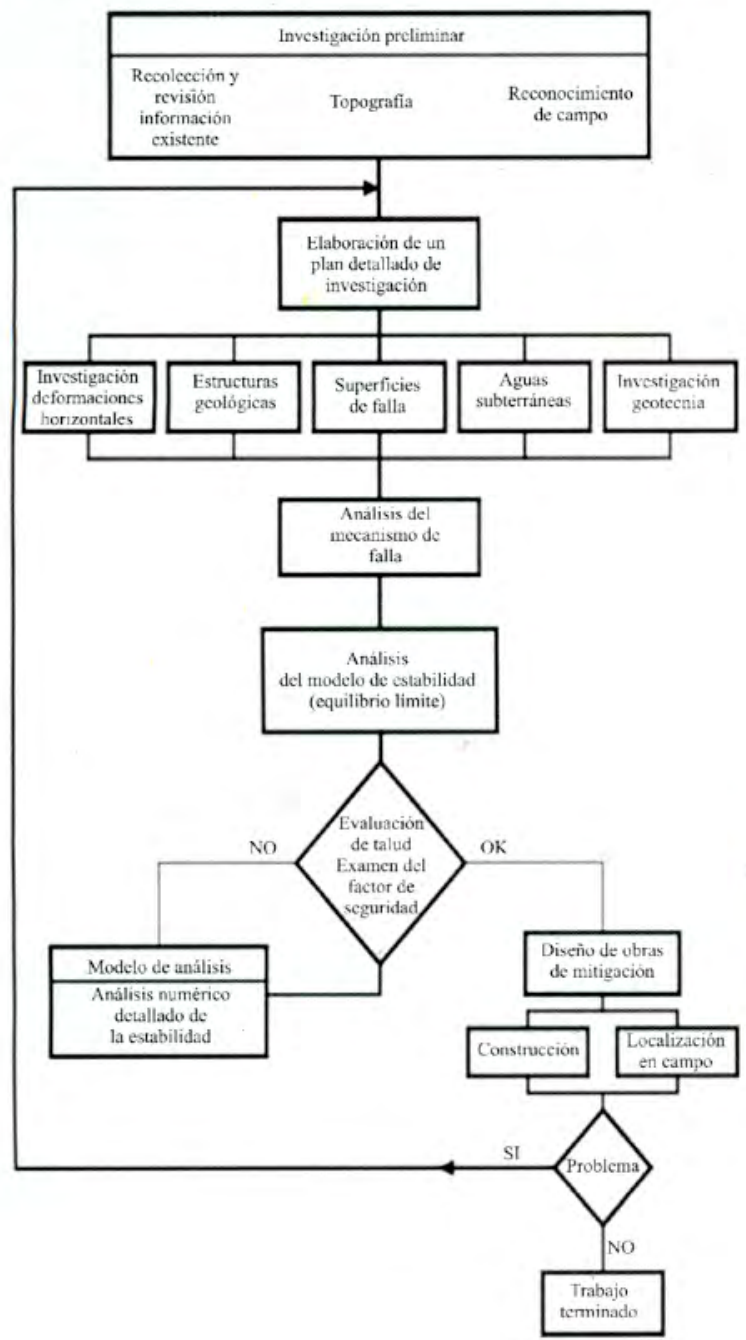

Figura N`02: Diagrama de flujo de metodología de investigación

\section{RESULTADOS Y DISCUSIÓN}

\section{a) Modelamiento del Talud Botadero $\mathrm{N}^{\circ} 05$}

Para el Modelamiento del Botadero $\mathrm{N}^{\circ} 05$ se ha utilizado el software Slide 5.0 de Rocscience Inc. y se ha obtenido los factores de seguridad siguientes:

\section{Bishop Simplified .- Ver Figura $\mathrm{N}^{\circ} 3$ Jambu Corrected .- Ver figura $\mathrm{N}^{\circ} 4$ Spencer .- Ver figura $\mathrm{N}^{\circ} 5$}

\section{b) Resultado del análisis}

Tabla $N^{\circ}$ 1: Factor de seguridad

\begin{tabular}{lc}
\hline \multicolumn{1}{c}{ Métodos } & Factor de Seguridad (F S) \\
\hline Bishop Simplified & 0.488 \\
Jambu Corrected & 0.397 \\
Spencer & 0.573 \\
Gle/Morgenstern & 0.636 \\
\hline
\end{tabular}

\section{c) Monitoreo geotécnico del botadero $\mathrm{N}^{\mathrm{0}} 05$ (Operación de Campo)}

Véase Tabla $\mathrm{N}^{\circ} 2$.

\section{d) Prevención y estabilizaciónes talud}

Una vez estudiado el talud, definidos los niveles de amenaza y riesgo, el mecanismo de falla y analizados los factores de equilibrio, se puede pasar al objetivo final que es el diseño del sistema de prevención control o estabilización.

Existen varias formas de enfocar y resolver cada problema específico y la metodología que se requiere emplear depende de una serie de factores técnicos, sociales, económicos, políticos; con una gran cantidad de variables en el espacio y en el tiempo.

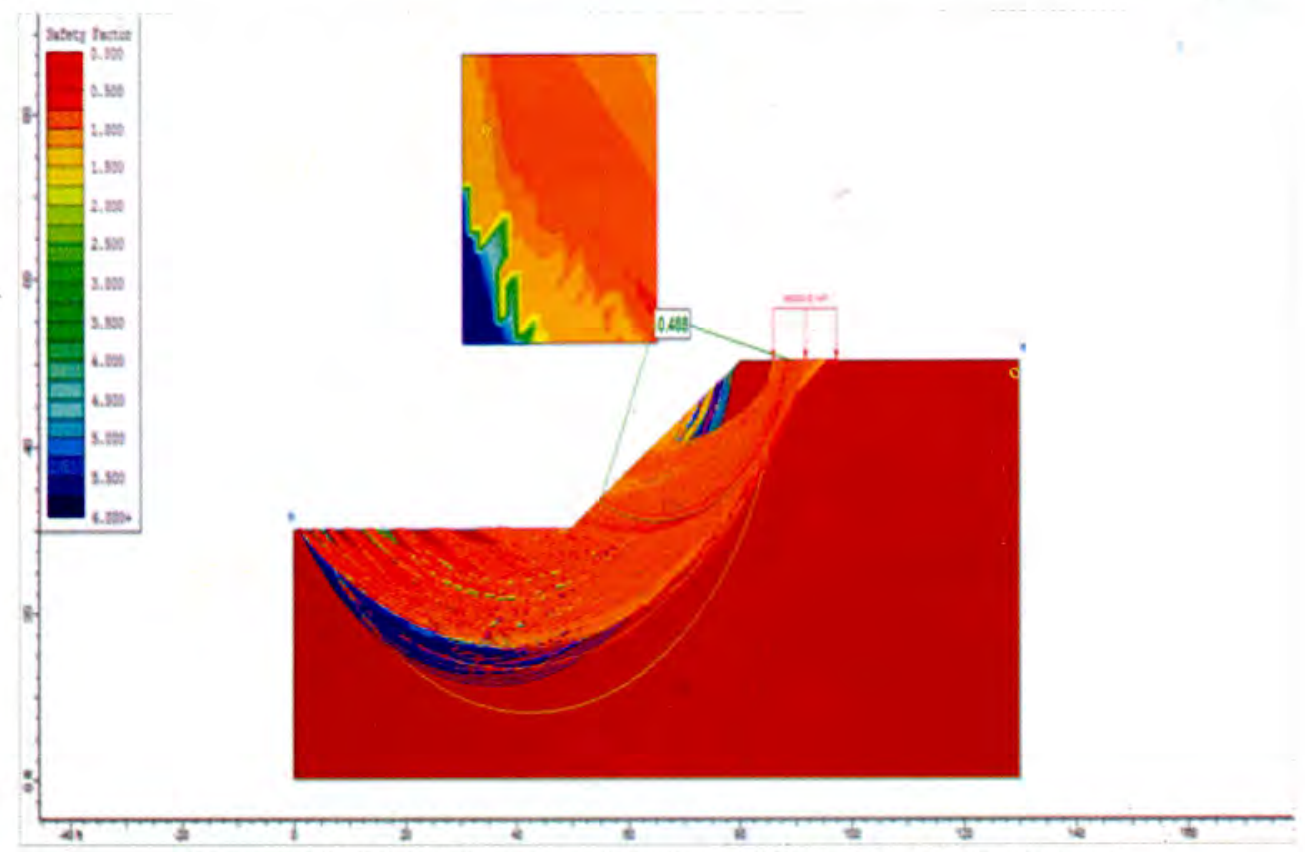

Figura $\mathrm{N}^{\mathbf{0}} \mathbf{3}$ :Modelamiento del talud del Botadero $\mathrm{N}^{\circ} 05$ (Bishop). 
Tabla $N^{\circ}$ 2: Rangos de desplazamientos totales y Velocidades Incrementales actuales, obtenidas mediante monitoreo Geotécnico del Botadero $\mathrm{N}^{\circ} 05$

\begin{tabular}{cccccc}
\hline Prisma & Ubicación en el Botadero $\mathrm{N}^{\circ} \mathbf{5}$ & Tendencia & $\begin{array}{c}\text { Plunge } \\
\text { Inclinación }\end{array}$ & $\begin{array}{c}\text { Desplazamiento } \\
\text { Total (m) }\end{array}$ & $\begin{array}{c}\text { Velocidad } \\
\text { Incremental } \\
\text { (cm/día) }\end{array}$ \\
\hline $\mathbf{5 0 - 1}$ & Carretera de Acceso a Mina & $\mathrm{N} 22^{\circ} \mathrm{W}$ & $-50^{\circ}$ & 3.4 & 0.32 \\
$\mathbf{5 0 - 2}$ & Carretera de Acceso a Mina & $\mathrm{N} 45^{\circ} \mathrm{W}$ & $-54^{\circ}$ & 8.6 & 1.48 \\
$\mathbf{5 0 - 3}$ & Deformación en Falla 3 Ichupampa & $\mathrm{N} 62^{\circ} \mathrm{W}$ & $-10^{\circ}$ & 15.0 & 2.90 \\
$\mathbf{5 0 - 4}$ & Zonas de Deformación & $\mathrm{N} 59^{\circ} \mathrm{W}$ & $-23^{\circ}$ & 17.4 & 3.09 \\
$\mathbf{5 0 - 5}$ & Zonas de Deformación & $\mathrm{N} 60^{\circ} \mathrm{W}$ & $-14^{\circ}$ & 22.1 & 3.90 \\
\hline
\end{tabular}

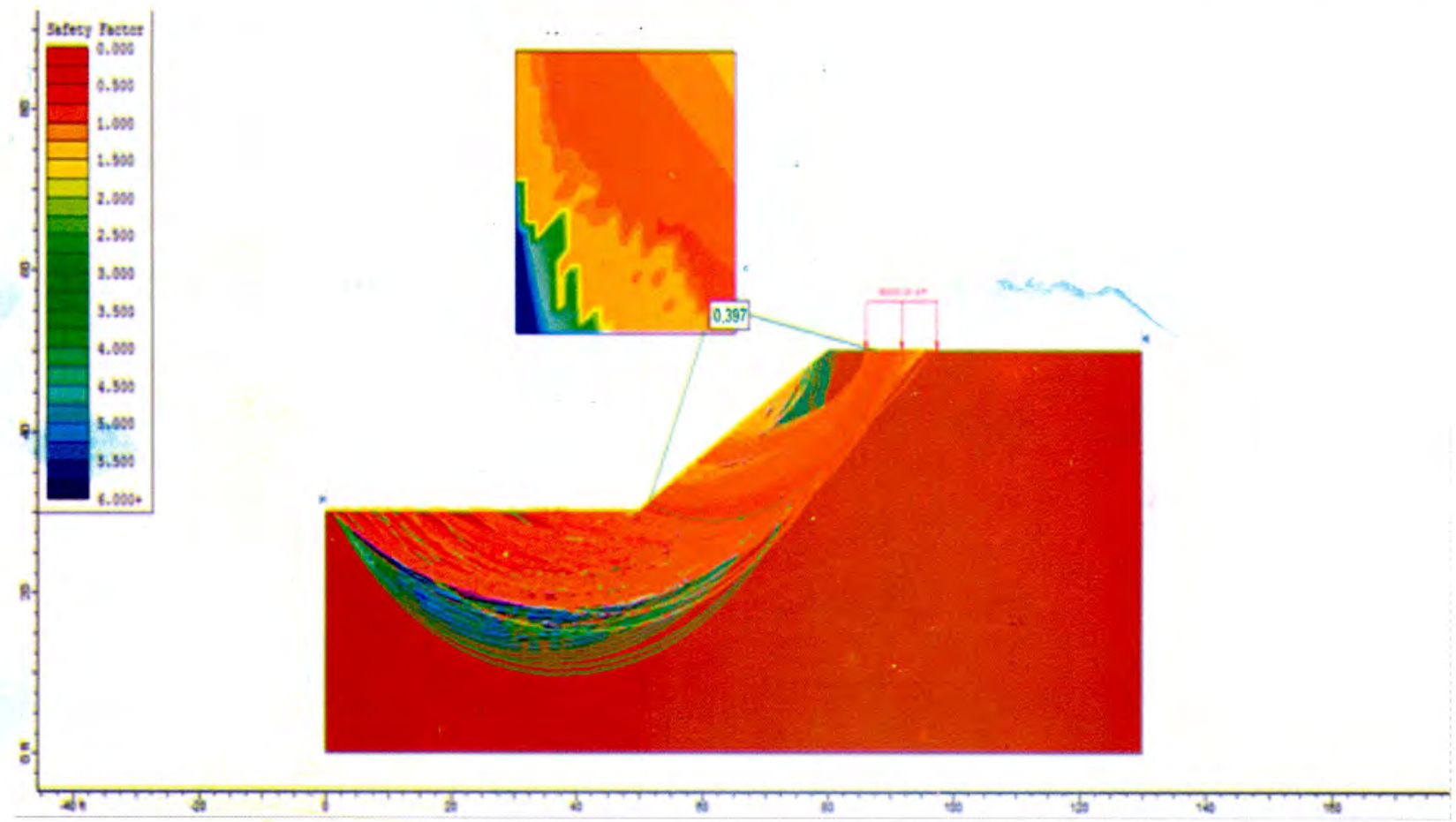

Figura $\mathrm{N}^{\circ} 04$ : Modelamiento del talud del Botadero $\mathrm{N}^{\circ} 05$ (Jambu).

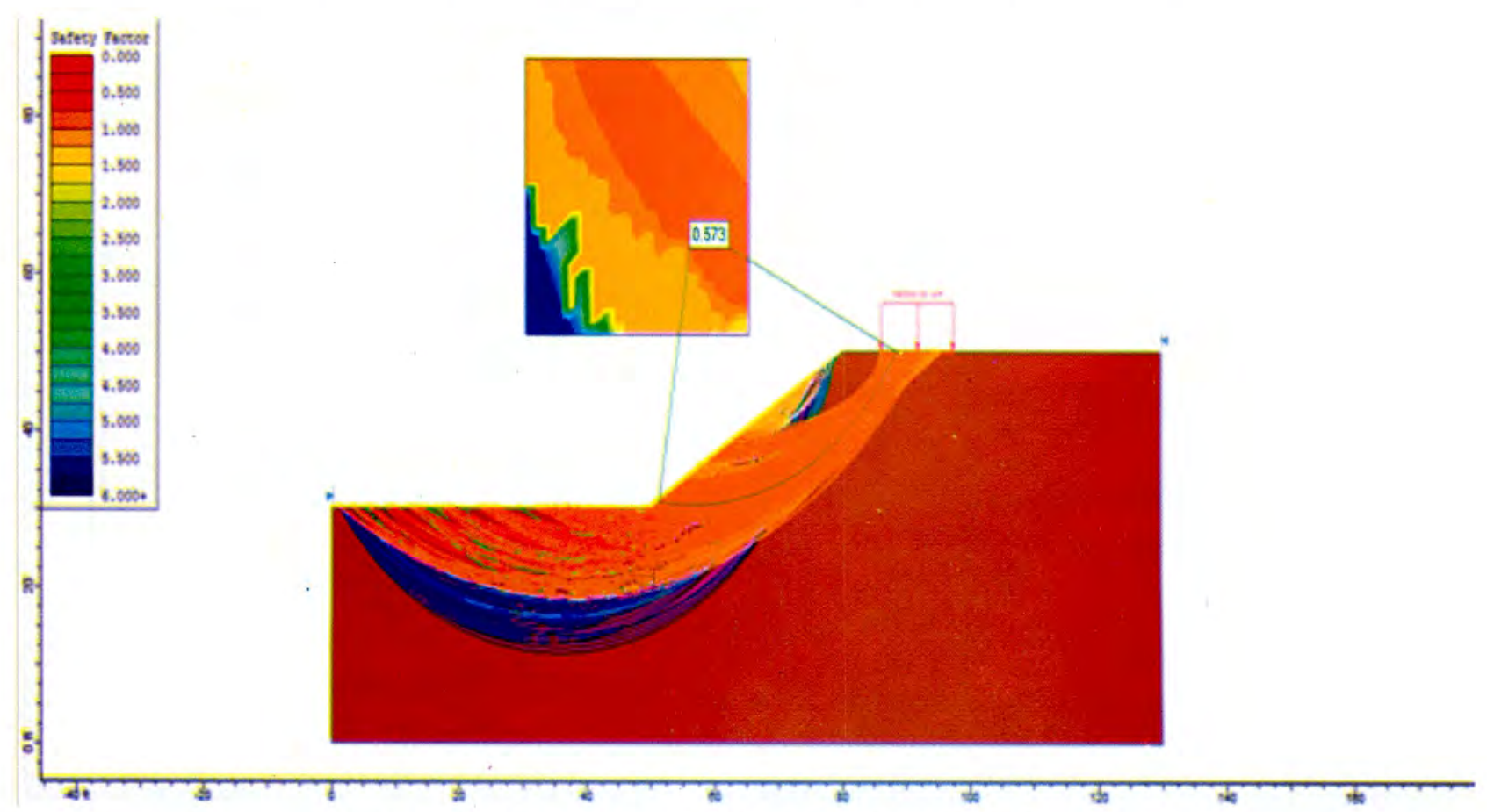

Figura No5: Modelamiento del Talud Botadero $N^{\circ} 05$ (Spencer). 


\section{CONCLUSIONES}

Litológica y estructuralmente es una zona compleja, con diferentes áreas de debilidad (Deslizamientos Antiguos y Fallas Geológicas) y su base está conformada de rocas volcánicos y intrusivos como Traquitas, Vitrofiro, Toba Salmón, Conglomerado Basal y Material Coluvial que influyen directamente en el proceso de deformación y en la estabilidad de los botaderos.

El Botadero $\mathrm{N}^{\circ} 05$ presenta un comportamiento completamente anormal, donde los procesos de deformación presentan una tendencia incremental (Fase Progresiva), que es reflejado con asentamientos mayores en las zonas de deformación Ichupampa (Desplazamientos total, Horizontal y Vertical), de 2.9, 3.09 y $3.9 \mathrm{~cm}$./dia. Que según las tablas de Varnes (1996) no es muy peligroso pero hay que tener mucho cuidado.

El análisis por Modelamiento utilizando Slide 5.0 de Rocscience arrojo los siguientes valores de F.S como: Bishop Sinplified (0.488), Jambu Corrected (0.397), Spencer (0.573), Gle/Morgenstern (0.636), lo que indica que el F.S. es inferior a 01 , el talud es inestable.

En el Modelamiento se ha aplicado Geotextiles como sostenimiento esto demuestra que el F.S. se eleva de 0.488 a 0.675 .

\section{REFERENCIAS BIBLIOGRÁFICAS}

Abramson L.W. (1996). "Engineering Geology Principles" Slopes stability and stabilizacion methods. Wiley interscience pp. 60-106.
Beer and Hegg (1992). "Landslide Stabilization at Ancona, Italy, by deep drainage Wells" Proceedings of the sixth internacional symposium on Landslides, Christchurch, pp. 663-670.

Brand (1985). "Glossary of Geology" American Geological Institute, falls Church. Va. 751 pp.

Corominas (1992) "Colluvium and Talus" Landslide investigation and mitigation special report 247 , Nacional research council pp. 5225-554

Choi, E. C.C (1977). "Seepage around Horizontal drains - two and three dimensional finite element análisis" Hong Kong Engineer, vol. $5 \mathrm{~N}^{\circ} 9$ pp. $35-39$.

Hoek and Brown (1988). "Excavaciones Sbterraneas en Roca" Rieview en Ingenieria Geologica pp. 1045

Kenney T.C. (1977). "Design of Horizontal Dains for Soil Slopes" Journal of the Geotechnical Engineering Division ASCE vol. 103 pp. 1311 - 1323.

\section{Correspondencia:}

Carlos Huisa Ccori

Ciudad Universitaria Fundo "Los Granados"

Av. Miraflores s/n. Tacna. Perú.

chuisac@unjbg.edu.pe 